\title{
BMJ Open Health-related quality of life in residents aged 18 years and older with and without disease: findings from the First Provincial Health Services Survey of Hunan, China
}

Xin Deng, ${ }^{1}$ Peng Dong, ${ }^{2}$ Lingling Zhang, ${ }^{3}$ Danping Tian, ${ }^{4}$ Lin Zhang, ${ }^{5}$ Wei Zhang, ${ }^{5}$ $\mathrm{Li} \mathrm{Li},{ }^{5}$ Jing Deng, ${ }^{5}$ Peishan Ning, ${ }^{5}$ Guoqing $\mathrm{Hu}^{5}$

To cite: Deng X, Dong $P$, Zhang L, et al. Health-related quality of life in residents aged 18 years and older with and without disease: findings from the First Provincial Health Services Survey of Hunan, China. BMJ Open 2017;7:e015880. doi:10.1136/ bmjopen-2017-015880

- Prepublication history for this paper is available online. To view these files please visit the journal online (http://dx.doi. org/10.1136/bmjopen-2017015880).

$\mathrm{XD}$ and $\mathrm{PD}$ contributed equally.

Received 6 January 2017

Revised 17 July 2017

Accepted 21 July 2017

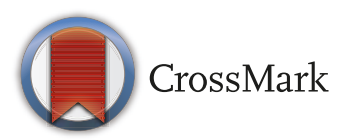

For numbered affiliations see end of article.

Correspondence to

Dr Guoging $\mathrm{Hu}$;

huguoging009@gmail.com

\section{ABSTRACT}

Objective Published research has not considered acute diseases and injuries in assessing the impact of varying disease counts on health-related quality of life (HRQOL). We used Chinese value sets of EQ-5D-3L to examine the relationship between the number of diseases individuals had (including chronic diseases, acute diseases and injuries) and their HRQoL.

Methods A total of 19387 individuals aged 18 years and older were included in the study. Using data from the First Provincial Health Services Survey of Hunan, China, HRQoL was assessed with the EQ-5D-3L scale, a standardized instrument developed by the EuroQoL group. The EQ-5D$3 \mathrm{~L}$ utility score was calculated using the Chinese EQ-5D$3 \mathrm{~L}$ value set. This survey coded disease using the list of 133 conditions that was defined by the First Provincial Health Services Survey of Hunan, China, based on the 10th International Classification of Diseases. 126 conditions were disease-related and were therefore included in data analysis.

Results 0 15245 respondents, urban residents and male constituted $53.0 \%$ and $48.2 \%$, respectively. $19.3 \%$ of respondents had one disease and $5.0 \%$ had at least two diseases. Of the five dimensions of the EQ-5D-3L, the pain/discomfort dimension had the highest proportion of moderate or serious problems among the respondents (14.4\%, $95 \% \mathrm{Cl} 10.5 \%$ to $18.2 \%)$. The average Visual Analogue Scale (VAS) score and utility score were 78.0 (95\% Cl 76.9 to 79.1 ) and 0.958 (95\% Cl 0.946 to 0.970 ), respectively. Residents with 1 and $\geq 2$ diseases had higher proportions of moderate or serious problems in five dimensions of the $E Q-5 D-3 L$ scale during the previous 2 weeks than those without disease after controlling for location (urban/rural), sex, age, education level and household income, respectively (adjusted ORs: 3.1-3.7 and 4.4-6.6, respectively). The mean of the EQ VAS score was 8.4 and 13.6 points lower in respondents with 1 and $\geq 2$ diseases than in respondents without disease; the corresponding mean score difference was 0.048 and 0.086 in EQ-5D-3L utility score. Disease-specific analyses were not conducted due to the inadequacy of sample size. Conclusions The HRQoL of residents aged 18 years and older declines distinctly as the number of diseases increases. Actions should be taken to improve the HRQD

\section{Strengths and limitations of this study}

- Large-scale population-based study examined the relationship between the number of diseases and health-related quality of life (HRQoL) by including acute diseases and injuries as well as chronic diseases.

- We used the latest population-based survey to report the HRQoL among 15245 Chinese respondents aged 18 years and older.

- The severity of diseases was not considered in this study due to the absence of relevant data.

- Disease-specific analysis was not performed because of sample size limitations.

of residents with multiple diseases in China (including acute diseases, chronic diseases and injuries).

\section{BACKGROUND}

Multimorbidity is defined as "the presence of more than one or multiple chronic or longterm diseases or conditions'. 'According to the Global Burden of Disease Study 2013, multiple morbidities are also common in all regions of the world among working-age adults (20-64 years). Reported rates of multimorbidity include $31.7 \%$ of individuals with five or more sequelae in developed countries, $37.9 \%$ in low-income/middle-income countries outside of sub-Saharan Africa and $61.6 \%$ in sub-Saharan Africa. ${ }^{2}$ Despite these data, patients with multimorbidity have not received the attention they deserve, especially for their health-related quality of life (HRQoL)..$^{34}$

Assessment of HRQoL among residents with varying disease counts can reflect the health outcomes of medical care service. ${ }^{5}$ Unfortunately, most published studies 
merely consider the association between the occurrence of disease and HRQoL, or the impact of complications and comorbidities of a certain disease on HRQoL. Huang et $a t^{6}$ reported adults with chronic conditions were more likely to have lower HRQoL in Heilongjiang, China, and Pan et al investigated the HRQoL among Chinese patients with type 2 diabetes with a variety of clinical characteristics. Very limited literature examines the impact of the number of diseases on the HRQoL of patients. Chin et $a l^{8}$ examined the impact of the combinations of three kinds of diseases (hypertension, diabetes and/or cardiovascular diseases) on the HRQoL in elderly patients in Korea. Tan $e t a t^{\dagger}$ and Andersson $e t$ $a l^{10}$ reported the impact of a number of chronic diseases on HRQoL. However, none includes acute diseases and injuries when assessing HRQoL among residents with varying disease counts. Considering that previous studies reported that acute diseases and injuries significantly affected HRQoL of persons, ${ }^{11-13}$ the exclusion of acute diseases and injuries may bias the results in assessing the association of multimorbidity with HRQoL. To include acute diseases and injuries as well as chronic diseases in data analysis will increase the knowledge of association of the number of diseases and injuries with HRQoL, and help identify the target populations with lower HRQoL that need care.

The First Provincial Health Household Interview Survey of Hunan, China, completed in 2013, collected health status of residents in the last 2 weeks (including acute diseases, chronic diseases and injuries) and EQ-5D-3L data, allowing us to examine the association of the number of diseases in the last 2 weeks, including acute diseases and injuries, with the HRQoL among residents aged 18 years and older.

The primary objective of this study was to examine whether the HRQoL varied with the number of diseases (including acute diseases, chronic diseases and injuries) among Chinese aged 18 years and older.

\section{METHODS AND MATERIALS \\ Data source}

Data came from the 2013 First Provincial Health Services Survey of Hunan, China. A total of 8400 households were sampled using multistage stratified cluster random sampling. The sample included 4200 rural households and 4200 urban households. Data were collected through face-to-face interviews, which were conducted by trained personnel using the First Provincial Health Services Survey questionnaire of Hunan, China. ${ }^{14}$

The Provincial Health and Family Planning Commission of Hunan Province (formerly called the Health Bureau of Hunan Province) administered the survey. A group of experts inspected the implementation of household surveys in all 14 sample counties (1-2 days per county), identifying any problems during the data collection and suggesting solutions.

\section{HRQoL measurement}

The EQ-5D-3L scale was used to measure the HRQoL of residents aged 18 years and older. The scale was developed by the EuroQol Group and is considered to be one of the best tools for measuring HRQoL. ${ }^{15-17}$ The Chinese version of EQ-5D-3L scale has good reliability and validity. ${ }^{18}$ The scale includes the EQ-5D-3L questionnaire and the EQ-5D-3L value set.

The EQ-5D-3L questionnaire contains a health description system and Visual Analogue Scale (VAS). For the health description system, the EQ-5D-3L adopts three options-no problems, moderate problems or serious problems-to reflect subjective health in each of five dimensions (mobility, self-care, activity, pain/discomfort and anxiety/depression). Detailed explanations of the three options are provided in the manual of EQ-5D-3L. ${ }^{15} 19$ Records were eliminated when someone else provided an answer on behalf of the respondent or when any domain was missing. ${ }^{15} 19$

The EQ-5D-3L values were calculated as an aggregated utility score of the five dimensions based on the Chinese time trade-off value set, which is a conversion weight from health utility measurements designed based on the HRQoL preferences of populations. ${ }^{20}$

\section{Diseases and covariates}

Disease in the last 2 weeks was defined as the occurrence of any of the following circumstances in the prior 14 days: (1) hospital visit; (2) receiving a medical treatment for disease or injury (eg, taking drugs, or receiving massage or hot compress); and (3) being off work or school, or in bed for more than 1 day for disease and injury. The classification of diseases was based on the list of 133 conditions defined by the First Provincial Health Services Survey of Hunan, China; this list divided all diseases and injuries into 133 kinds of diseases, signs, symptoms and abnormalities of 21 subgroups, based on the 10th International Classification of Diseases. ${ }^{14}$ Only 126 kinds of conditions were included in the analysis related to the number of diseases because seven conditions were judged not to be diseases, such as 'artificial abortion'. Of the 126 categories, only major causes of diseases were specified; many uncommon and relevant diseases (including acute and chronic diseases) that have relatively low morbidity rates were combined into a single category. Thus, we cannot clearly separate acute diseases from chronic diseases in our analysis. The field investigators collected information about disease according to the face-to-face interview instructions and classified each condition as one of 133 conditions based on the interviewee's answer or medical records. All investigators received standardised training before the survey, including training on the methods of disease classification and coding disease, face-to-face interview skills, and other relevant issues. This survey collected the information about diseases if the interviewee reported any disease during the previous 2 weeks.

Based on the relevant literature 89222 and the availability and reliability of demographic variables in the First 
Provincial Health Services Survey of Hunan, we chose to include the following covariates in our analysis: location (urban/rural), sex, age, education level and household income per capita. We divided the households equally into five categories based on the household income per capita in the last year for urban and rural areas separately: lowest (urban, <¥6667; rural, <¥3334); lower (urban, $¥ 6667-¥ 9999$; rural, ¥3334-¥4999); average (urban, ¥10 000-¥14 999; rural, ¥5000-¥7499); higher (urban, ¥15 000-¥23 999; rural, ¥7500-¥9999); and highest (urban, $\geq ¥ 24000$; rural, $\geq ¥ 10000$ ).

\section{Ethical approval}

All procedures performed in studies involving human participants were in accordance with the ethical standards of the institutional and/or national research committee and with the 1964 Helsinki Declaration and its later amendments or comparable ethical standards. The survey was organised by the Provincial Health and Family Planning Commission of Hunan Province, China. The data analysis was de-identified and was approved by the medical ethics committee of Central South University (XYGW-2016-18).

\section{Statistical analysis}

Complete case analysis was used for all analyses. Because very few residents reported 'serious problems' in all of the five dimensions, we combined the groups with 'moderate problems' and 'serious problems' into a single group; this decision followed previous publications also. ${ }^{5}$ Univariate and multiple logistic regression based on sampling weights were performed to examine the impact of the number of diseases on the occurrence of 'moderate or serious problems' in mobility, self-care, activity, pain/ discomfort and anxiety/depression.

$\mathrm{Utt}^{23}$ defined the 'ceiling effect' as occurring when the tests or scales are developed such that substantial proportions of individuals obtain either maximum or near-maximum scores and the true extent of their abilities cannot be determined. In our study, many participants answered every item with 'no problem', creating a situation where some VAS and utility scores censored at 100 and 1.0 and suggesting the presence of a 'ceiling effect'. As Austin $e$ t $a l^{24}$ did, we used Tobit regression to quantify the impact of some demographic characteristics and the number of diseases on the VAS score and utility score.

We changed the reference group of number of diseases in multivariate models from 'no disease' to ' 1 disease' to examine the statistical significance of trends in HRQoL across residents with 0,1 and $\geq 2$ diseases. $\chi^{2}$ test was performed to examine the distribution of number of diseases across age groups.

Statistical analyses were completed using SAS V.9.2 software and Stata V.11.0 software at the significance level of $\alpha=0.05$.

\begin{tabular}{|c|c|c|}
\hline Demographic variables & $\mathbf{N}$ & Proportion (\%) \\
\hline Total & 15245 & 100.0 \\
\hline \multicolumn{3}{|l|}{ Location } \\
\hline Urban & 8086 & 53.0 \\
\hline Rural & 7159 & 47.0 \\
\hline \multicolumn{3}{|l|}{ Sex } \\
\hline Male & 7351 & 48.2 \\
\hline Female & 7894 & 51.8 \\
\hline \multicolumn{3}{|l|}{ Age group } \\
\hline 18-44 years & 4902 & 32.2 \\
\hline $45-64$ years & 7225 & 47.4 \\
\hline$\geq 65$ years & 3118 & 20.5 \\
\hline \multicolumn{3}{|l|}{ Education } \\
\hline$\leq 6$ years & 6025 & 39.5 \\
\hline $7-12$ years & 7838 & 51.4 \\
\hline$\geq 13$ years & 1382 & 9.1 \\
\hline \multicolumn{3}{|c|}{ Household income per capita* } \\
\hline Lowest & 3007 & 19.8 \\
\hline Lower & 2139 & 14.1 \\
\hline Average & 3393 & 22.3 \\
\hline Higher & 2762 & 18.2 \\
\hline Highest & 3897 & 25.6 \\
\hline \multicolumn{3}{|l|}{ Number of diseases } \\
\hline 0 & 11715 & 75.7 \\
\hline 1 & 2785 & 19.3 \\
\hline$\geq 2$ & 745 & 5.0 \\
\hline
\end{tabular}

Note: Weighted proportion was calculated based on sampling weights.

*The incomes of some households were missing. Household income per capita included five categories: lowest (urban, $<¥ 6667$;

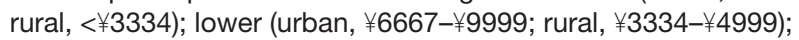
average (urban, $¥ 10000-¥ 14999$; rural, $¥ 5000-¥ 7499$ ); higher (urban, $¥ 15000-¥ 23999$; rural, $¥ 7500-¥ 9999$ ); and highest (urban, $\geq ¥ 24000$; rural, $\geq ¥ 10000$ ).

\section{RESULTS}

\section{Sample characteristics}

In total, 19387 individuals aged 18 years and older were included in the survey, and $78.6 \%$ of them completed the questions of EQ-5D-3L scale. The average age of respondents was 52.2 years old (SE: 0.55 years). Urban residents and male constituted $53.0 \%$ and $48.2 \%$ of study respondents, respectively. There were $39.5 \%$ of study subjects who reported primary education or lower ( $\leq 6$ years) and $51.4 \%$ who reported secondary education ( $7-12$ years) (table 1).

Of all respondents aged 18 years and older, $5.0 \%$ had two or more diseases, and $19.3 \%$ had one disease (figure 1). A $\mathrm{X}^{2}$ test showed that the proportions of residents having 1 and $\geq 2$ diseases were highest among the age group of 65 years and older $(33.7 \%$ and $10.5 \%, \mathrm{p}<0.05)$. 


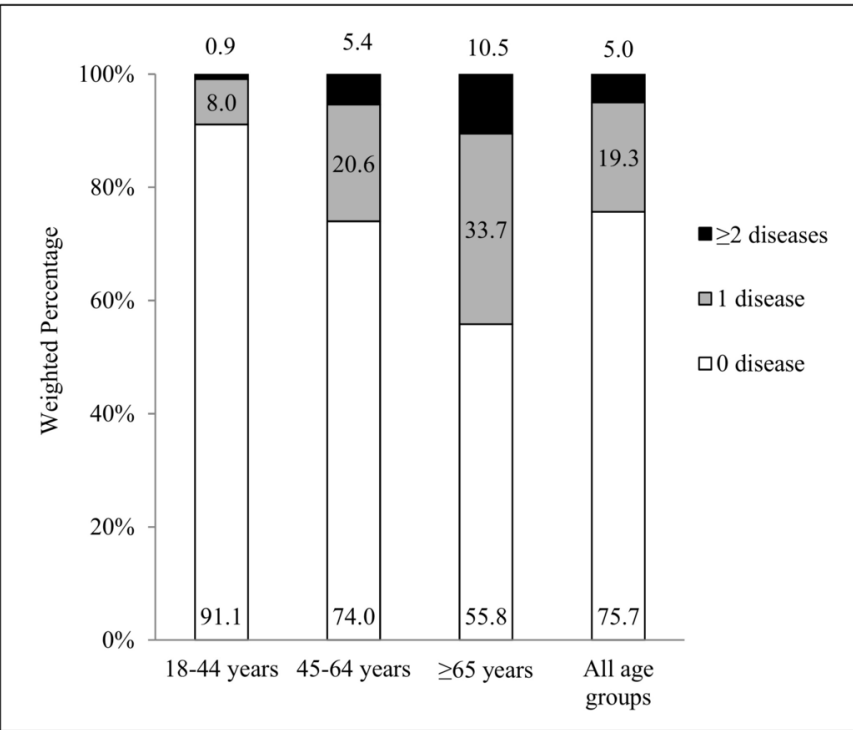

Figure 1 Distribution of the number of diseases by age group in Hunan, China, 2013.

\section{EQ-5D-3L in different demographic characteristics}

Residents had the highest proportion of moderate or serious problems in the pain/discomfort dimension (14.4\%, 95\% CI $10.5 \%$ to $18.2 \%$ ) (table 2 ). The average score of VAS was 78.0 (95\% CI 76.9 to 79.1 ). Univariate analysis showed that participants who were older, had lower levels of education, were from low-income households and had 1 and $\geq 2$ diseases had more problems and lower VAS scores.

\section{Number of diseases}

The results showed that the proportion of having problems in all five dimensions of EQ-5D-3L significantly increased as the number of diseases increased. Compared with residents without disease in the last 2 weeks, residents with 1 and $\geq 2$ diseases had higher proportions of moderate or serious problems in the five dimensions after controlling for location (urban/rural), sex, age group, education level and per capita household income, respectively (adjusted OR: mobility, 3.6 and 5.8; self-care, 3.3 and 4.4; usual activities, 3.7 and 6.6; pain/discomfort, 3.3 and 5.2; and anxiety/depression, 3.1 and 4.9) (table 3). Residents with $\geq 2$ diseases had higher proportions of moderate or serious problems compared with those with 1 disease (adjusted OR: mobility, 1.6; self-care, 1.4; usual activities, 1.8; pain/discomfort, 1.6; and anxiety/depression, 1.7) $(\mathrm{p}<0.05)$.

Of the 15245 respondents, 915 had a VAS score of 100 and 12432 had a utility score of 1 , indicating the presence of ceiling effects. Tobit regression revealed that the average EQ-VAS score declined by 8.4 and 13.6 points in residents with 1 and $\geq 2$ diseases compared with those without disease after adjusting for location, sex, age, education level and household income. Further, residents with $\geq 2$ diseases had higher average VAS score than those with 1 disease $(b=-5.7, \mathrm{p}<0.05)$. The average utility scores were 0.048 and 0.086 lower in residents with 1 and $\geq 2$ diseases compared with those without any disease, and the average utility scores were 0.048 lower in residents with $\geq 2$ diseases than in those with 1 disease $(\mathrm{p}<0.05)$ (table 3).

\section{DISCUSSION}

We report some of the first data suggesting that as the number of diseases (including acute diseases and injuries over the past 2 weeks, as well as chronic diseases) increased, the proportion of residents with moderate or serious problems for all five dimensions of quality of life significantly increased, and both the EQ-VAS score and utility score substantially declined. Our results strongly suggest that multimorbidity of disease was significantly associated with worse HRQoL. Given that $37.9 \%$ of adults aged 20-64 years report five or more sequelae in low-income/middle-income countries outside of sub-Saharan Africa, ${ }^{2}$ our results indicate this population may suffer from multimorbidity-associated reduced HRQoL. They deserve attention from government, international organisation and researchers. To respond to this potential health challenge and the corresponding health service need, appropriate medical services, such as personalised, patient-centred treatment programme that can improve the HRQoL of patients with multiple diseases, should be developed and disseminated worldwide. Such efforts would alleviate the severity of multimorbidity and avoid unwanted adverse implications that result from inappropriate or delayed care.

The proportions of residents having moderate or serious problems in the five dimensions and mean VAS score assessed in this study match very closely with reports from a previous study from China, ${ }^{9}$ but the utility score based on a Chinese value set is higher than the results of the study performed by Zhang et $a l^{25}$ that targeted residents aged 60 years and older $(0.958$ vs 0.860$)$. The differences in HRQoL between this study and previous publications may be due to differences in the study time and the study population. We found similar HRQoL differences across age, sex, education and household income groups, as Mielck et al reported. ${ }^{26}$ Specifically, we found that older residents, those with lower levels of education and those from low-income households also reported poorer HRQoL.

In addition, we observed different regression coefficients $(b)$ for the VAS score and the EQ-5D-3L utility score. The difference may be due to measurement methods. The EQ VAS score measures the overall self-report health status of the respondent, whereas the utility score is converted from a health state based on the reporting results in the five dimensions of the EQ-5D-3L and the according value set. ${ }^{27}$

This study has several limitations. First, because the severity of diseases was not quantitatively assessed in this survey, we cannot eliminate its influence on the results. Empirically, the severity and number of diseases and injuries may represent two distinct factors that influence 


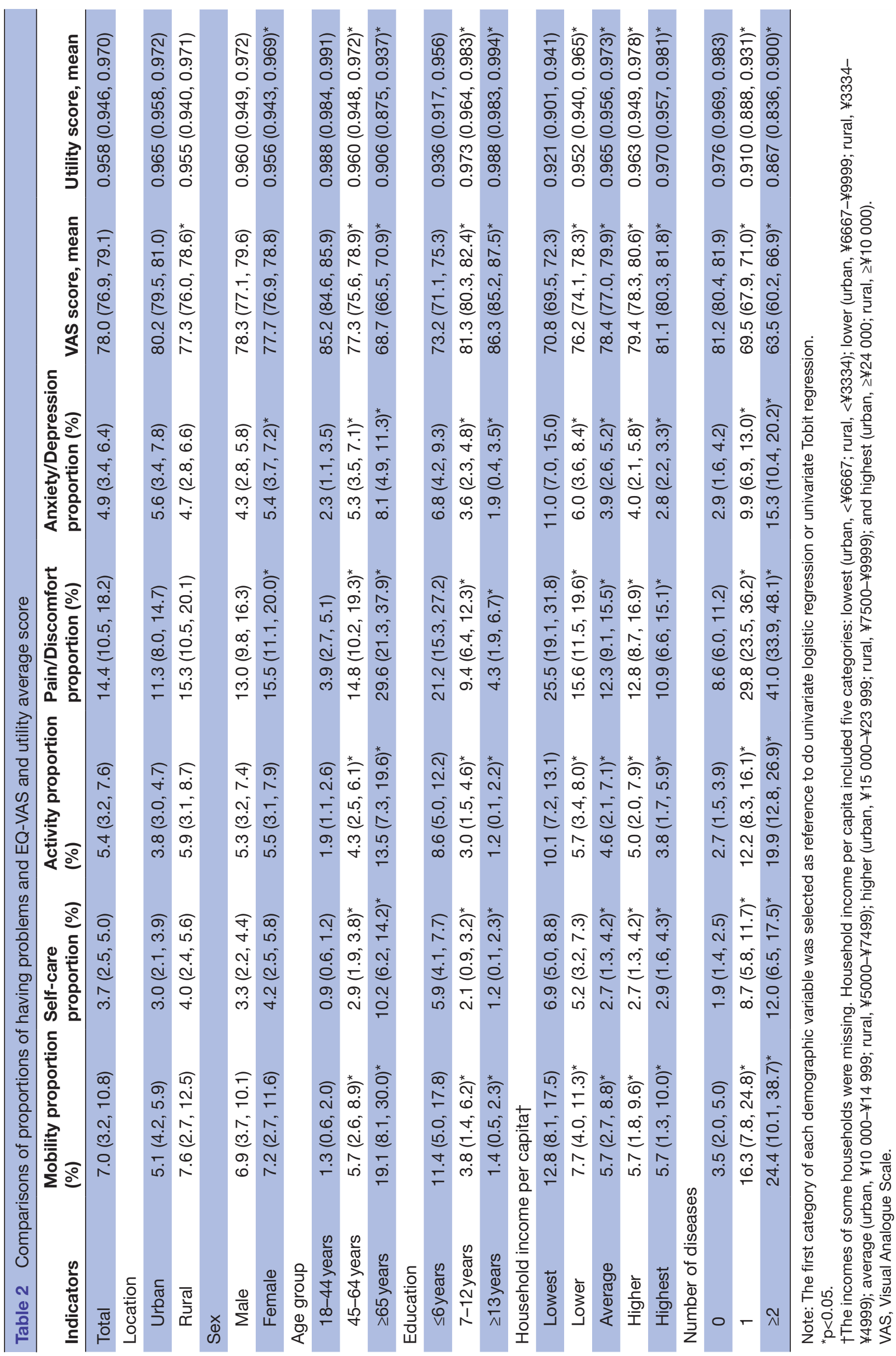

을 


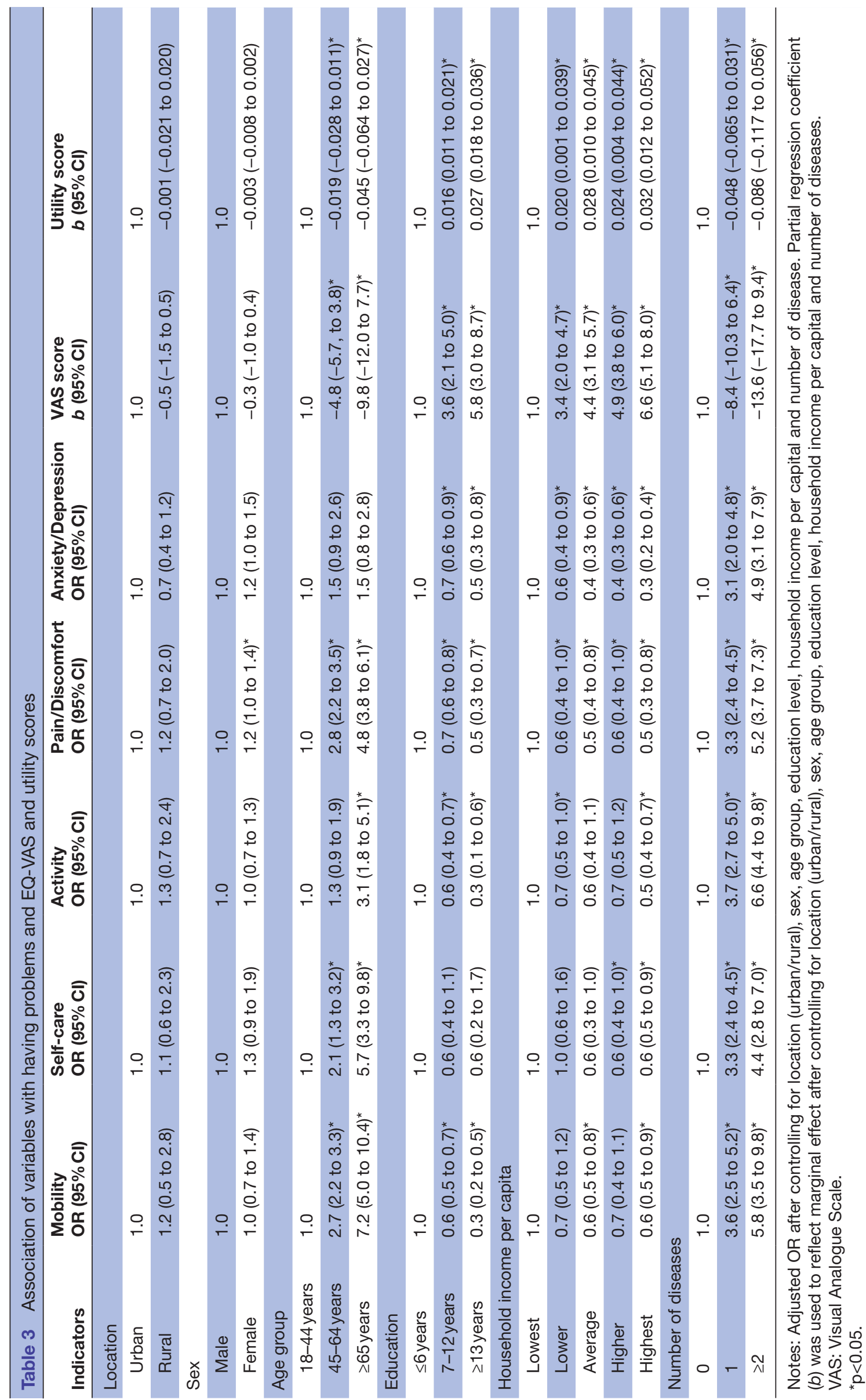


HRQoL, and further studies are warranted to explore the impact of disease severity on HRQoL in this population. Second, we did not conduct disease-specific analysis, nor did we compare results between acute diseases, chronic diseases and injuries. We omitted these analyses due to the inadequacy of sample size and the fact that we could not easily divide the types of diseases/injuries based on the data available in the survey. This restricts us from assessing disease-specific impact on HRQoL and exploring the precise quantitative relationship between the number of diseases and HRQoL. In the future, large-sample research should be conducted to continue to explore the unresolved issues and generate evidence for policy makers on how to take actions to improve the HRQoL of residents with disease. Third, we did not analyse costs of disease because data on costs were unavailable. Moreover, about $20 \%$ of respondents had missing values for the EQ-5D-3L survey questions. Further analysis indicates that the distribution of all demographic variables for sample with missing HRQoL value is generally consistent with that for sample with complete HRQoL value except for location (urban/rural) (not shown here). In light of differences in three key indicators (proportions of having problems, VAS score, and utility score) are somewhat similar between urban and rural respondents, we believe that the missing value data would not substantially influence the results.

\section{CONCLUSIONS}

In this study, HRQoL markedly declined among residents aged 18 years and older as the number of diseases (including acute diseases and injuries and chronic diseases) over the past 2 weeks increased. Attention should be paid to multimorbidity from acute diseases and injuries as well as chronic diseases by healthcare providers and policy-makers. Large-sample studies are needed to assess the impacts of severity of disease and kinds of disease on HRQoL in addition to the effect of the number of diseases on HRQoL, thus generating prioritised policy actions to improve HRQoL of residents.

\section{Author affiliations \\ ${ }^{1}$ Xiangya Hospital, Central South University, Changsha, China \\ ${ }^{2}$ Department of Health Management, Peking University Health Science Center, \\ Beijing, China \\ ${ }^{3}$ Department of Public Health Sciences, Clemson University, Clemson, USA ${ }^{4}$ Division of Scientific Research, Hunan Province Children's Hospital, Changsha, Hunan, China \\ ${ }^{5}$ Department of Epidemiology and Health Statistics, Xiangya School of Public Health, Central South University, Changsha, China}

Contributors XD and PD worked collaboratively to implement the data analysis, interpreted the findings and drafted the manuscript. GH conceived of the research idea, supervised the research and finalised the manuscript. All other authors contributed to the critical review of the methodology of the study and were involved in all the drafts and revisions of the paper. All authors approved the final manuscript.

Competing interests None declared.

Provenance and peer review Not commissioned; externally peer reviewed.
Data sharing statement The data sets generated and analysed during the current study are not publicly available as we are required to comply with the request of Provincial Health and Family Planning Commission of Hunan Province, China, which supported the study, to keep the original data confidential.

Open Access This is an Open Access article distributed in accordance with the Creative Commons Attribution Non Commercial (CC BY-NC 4.0) license, which permits others to distribute, remix, adapt, build upon this work non-commercially, and license their derivative works on different terms, provided the original work is properly cited and the use is non-commercial. See: http://creativecommons.org/ licenses/by-nc/4.0/

(C) Article author(s) (or their employer(s) unless otherwise stated in the text of the article) 2017. All rights reserved. No commercial use is permitted unless otherwise expressly granted.

\section{REFERENCES}

1. Almirall J, Fortin M. The coexistence of terms to describe the presence of multiple concurrent diseases. J Comor 2013;3:4-9.

2. Global Burden of Disease Study 2013 Collaborators. Global, regional, and national incidence, prevalence, and years lived with disability for 301 acute and chronic diseases and injuries in 188 countries, 19902013: a systematic analysis for the Global Burden of Disease Study 2013. Lancet 2015;386:743-800.

3. Wolff JL, Starfield B, Anderson G. Prevalence, expenditures, and complications of multiple chronic conditions in the elderly. Arch Intern Med 2002;162:2269-76.

4. Starfield B, Shi L, Macinko J. Contribution of primary care to health systems and health. Milbank Q 2005;83:457-502.

5. Heyworth IT, Hazell ML, Linehan MF, et al. How do common chronic conditions affect health-related quality of life? Br J Gen Pract 2009;59:353-8.

6. Huang W, Yu H, Liu C, et al. Assessing health-related quality of life of chinese adults in heilongjiang using EQ-5D-3L. Int J Environ Res Public Health 2017;14:224.

7. Pan CW, Sun HP, Zhou HJ, et al. Valuing health-related quality of life in type 2 diabetes patients in China. Med Decis Making 2016;36:234-41.

8. Chin YR, Lee IS, Lee HY. Effects of Hhrtension, diabetes, and/or cardiovascular disease on health-related quality of life in elderly Korean Individuals: a population-based cross-sectional survey. Asian Nurs Res 2014;8:267-73.

9. Tan Z, Liang Y, Liu S, et al. Health-related quality of life as measured with EQ-5D among populations with and without specific chronic conditions: a population-based survey in Shaanxi Province, China. PLoS One 2013;8:e65958.

10. Andersson LB, Marcusson J, Wressle E. Health-related quality of life and activities of daily living in 85-year-olds in Sweden. Health Soc Care Community 2014;22:368-74.

11. Hnyp IY. Assessment of functional status and quality of life of students after acute respiratory viral diseases. Pedagogics, Psychology, Medical-Biological Problems of Physical Training and Sports 2015;3:10-14.

12. Lee SJ, Kim HT, Ho VT, et al. Quality of life associated with acute and chronic graft-versus-host disease. Bone Marrow Transplant 2006;38:305-10.

13. Gauffin E, Öster C, Sjöberg F, et al. Health-related quality of life (EQ-5D) early after injury predicts long-term pain after burn. Burns 2016;42:1781-8.

14. Center for Health Propaganda and Education, Health and Family Planning Commission of Hunan Province, China. 2013. Manual of the first provincial health household interview survey of hunan province. Changsha, China: Center for Health Propaganda and Education, Health and Family Planning Commission of Hunan Province.

15. EuroQol Group. EuroQol-a new facility for the measurement of health-related quality of life. Health Policy 1990;16:199-208.

16. Fitzpatrick R, Fletcher A, Gore S, et al. Quality of life measures in health care. I: applications and issues in assessment. BMJ 1992;305:1074-7.

17. Fletcher A, Gore S, Jones D, et al. Quality of life measures in health care. II: design, analysis, and interpretation. BMJ 1992;305:1145-8.

18. Wang $\mathrm{H}$, Kindig DA, Mullahy J. Variation in Chinese population health related quality of life: results from a EuroQol study in Beijing, China. Qual Life Res 2005;14:119-32.

19. Zhang YG, Xu L. Research on the health related quality of life of Chinese residents. Medicine and Society 2013;6:82-4.

20. Liu GG, Wu H, Li M, et al. Chinese time trade-off values for EQ-5D health states. Value Health 2014;17:597-604. 
21. He M, Wang Q, Zhu S, et al. Health-related quality of life of doctors and nurses in China: findings based on the latest open-access data. Qual Life Res 2012;21:1727-30.

22. Hu G, Rao K, Baker SP. Non-fatal injuries among Chinese aged 65 years and older: findings from the Fourth National Health Services Survey. Inj Prev 2010;16:230-4.

23. Uttl B. Measurement of individual differences: lessons from memory assessment in research and clinical practice. Psychol Sci 2005;16:460-7.

24. Austin PC, Escobar M, Kopec JA. The use of the Tobit model for analyzing measures of health status. Qual Life Res 2000;9:901-10.
25 Zhang T, Shi W, Huang Z, et al. Influence of culture, residential segregation and socioeconomic development on rural elderly healthrelated quality of life in Guangxi, China. Health Qual Life Outcomes 2016;14:98.

26 Mielck A, Reitmeir P, Vogelmann M, et al. Impact of educational level on health-related quality of life (HRQL): results from Germany based on the EuroQol 5D (EQ-5D). Eur J Public Health 2013;23:45-9.

27 Reenee MV, Oppe M. EQ-5D-3L User Guide: Basic information on how to use the EQ-5D-3L instrument (Version 5.1). The Netherlands: EuroQol Research Foundation, 2015. 